\title{
Compound-Specific Toxicities Detected in CFU-GM, Rat Kidney NRK Cells, Rat Bladder RBLAK Cells, and Rat Liver Slices following Batracylin or N-Acetyl Batracylin Exposure
}

\author{
Facundo M. Cutuli and Holger P. Behrsing \\ Lab of Investigative Toxicology, Applied/Developmental Research Directorate, Leidos Biomedical Research, Inc., \\ Frederick National Lab for Cancer Research, Frederick, MD 21702, USA
}

Correspondence should be addressed to Holger P. Behrsing; behrsing@advancedinvitro.com

Received 30 April 2014; Revised 3 July 2014; Accepted 4 August 2014; Published 8 September 2014

Academic Editor: Rosa Busquets

Copyright (C) 2014 F. M. Cutuli and H. P. Behrsing. This is an open access article distributed under the Creative Commons Attribution License, which permits unrestricted use, distribution, and reproduction in any medium, provided the original work is properly cited.

\begin{abstract}
The investigational anticancer agent batracylin (BAT; 8-aminoisoindolo [1,2-b]quinazolin-10(12H)-one; NSC320846) causes $\gamma$ $\mathrm{H} 2 \mathrm{AX}$ foci development in exposed tumor cells and has demonstrated activity against solid tumors and adriamycin-resistant leukemia. Reports indicate BAT has wide interspecies variation of adverse effects, including myelosuppression, kidney, bladder, and liver damage, including biliary hyperplasia. The effects of BAT and its metabolite N-acetyl batracylin (NAB) were evaluated in the CFU-GM bone marrow toxicity assay, rat kidney (NRK) cells, bladder epithelial (RBLAK) cells, and rat precision cut liver slices (PCLS). Exposure effects were evaluated biochemically and histologically. Human, dog, and rat exhibited similar CFU-GM IC90 values for BAT $(21-29 \mu \mathrm{M})$. The ATP assay and $\gamma$-H2AX staining showed time- and concentration-dependent toxicity in RBLAK (more severe than NRK at $<72 \mathrm{hr}$ ) NRK and cells (IC50 $<20 \mu \mathrm{M}$ after $96 \mathrm{hr}$ BAT exposure). BAT $(5 \mu \mathrm{M}$ and $25 \mu \mathrm{M})$ caused biochemical and histology changes to PCLS by day 3 and $25 \mu \mathrm{M}$ produced centrilobular hepatotoxicity. NAB ( $\leq 5 \mu \mathrm{M})$ produced no toxicity in CFU-GM, NRK, or RBLAK cells. However, both BAT and NAB caused biliary epithelial cell proliferation in PCLS. Our studies demonstrated species similarities in sensitivity to BAT-induced myelosuppression, and implicate the metabolite NAB in biliary hyperplasia.
\end{abstract}

\section{Introduction}

Batracylin, 8-aminoisoindolo[1, 2-b]quinazolin-12(10H)-one (BAT), is a heterocyclic arylamine first synthesized by Bayer AG, Leverkusen, Germany, and was acquired through the $\mathrm{NCI}$ as a potential pharmacodynamics agent for development to clinical trial based on its activity in the refractory colon adenocarcinoma 38 model [1]. It has demonstrated activity against solid tumors and adriamycin-resistant leukemia $[1,2]$ and has been evaluated preclinically in multiple species. Studies have confirmed BAT induces ATP-independent topoisomerase II (Topo II) inhibition [3, 4] and subsequent DNA strand breaks that can be detected using the $\gamma$-H2AX marker [5].
IND-directed toxicology studies of BAT were conducted in 3 mammalian species (1 dose, p.o., per day for 9 consecutive days). The variation in tolerated doses across species (mice, rats, and dogs) yielded an MTD range of at least 60-fold [6]. The major toxicities were myelosuppression in all species. Variable amounts of renal, hepatic, testicular, ovarian, gastrointestinal, and lethargy were found in the various species tested, and the rat was found to be the most sensitive for adverse effects. For rats, the severe toxic effects occurred at doses as low as 1/30 MELD10 of mice and included bone marrow atrophy; gastrointestinal atrophy, necrosis, degeneration, and inflammation; renal tubular necrosis; seminiferous tubular necrosis in males; ovarian degeneration in females; and bile duct hyperplasia. Pharmacology studies of batracylin in 
vivo and in vitro indicate that rats extensively acetylate batracylin to produce $\mathrm{N}$-acetyl batracylin (NAB) [7-9]. Mice produce relatively small amounts and dogs do not generate this metabolite. Calculation of plasma AUC values for NAB following oral administration of batracylin $\left(590 \mathrm{mg} / \mathrm{m}^{2}\right)$ to mice and rats revealed that systemic levels of NAB in the rat were 8.8 times greater than in the mouse [10]. Published data infers that NAB is a toxic metabolite $[7,8,10]$, but studies where $\mathrm{NAB}$ was administrated to rats (p.o.) indicate absence of toxicity, and it was speculated to be due to the poor bioavailability with this route of dosing. Given that $\mathrm{N}$-acetylation has been studied in human livers and it was found that human tissue can have varying rates of acetylation capability [9], further evaluation of potential NAB toxicity is warranted.

A variety of cell/tissue types and assays were chosen to evaluate BAT and NAB. To address reported myelosuppression, three species (rat, dog, and human) were chosen for the colony forming unit, granulocyte macrophage (CFU-GM) bone marrow toxicity assay. Previous success and reproducibility of the validated CFU-GM assay led to an international effort, led by the European Center for Validation of Alternative Methods (ECVAM), to establish a standard operating procedure, validate the assay SOP via multilaboratory evaluation, and propose its use as an alternative to a second species toxicology evaluation [11-16]. For reported kidney and bladder toxicities (the latter detected in clinical studies), NRK-52E (NRK) and RBLAK-WIS (RBLAK) cells, respectively, were evaluated using the ATP viability assay following exposure to BAT or NAB. NRK cells have been used as cell models of kidney that includes chemotherapeutic induced toxicity $[17,18]$. RBLAK cells (bladder progenitor cells derived from Wistar rat bladders) have previously been used as a model for bladder epithelium in pharmaceutical research (personal communication with Peter Girling, CEO of CELLnTEC). To evaluate hepatobiliary toxicities reported, studies using male F344 rat precision-cut liver slices (PCLS) were conducted and toxicity evaluated using biochemical and histological means. The use of organotypic PCLS for detection of adverse effects related to exposure to drugs and/or compounds inducing biliary hyperplasia has previously been reported [19-23].

\section{Materials and Methods}

2.1. Materials. Batracylin (NSC320846) and N-acetyl batracylin (NSC611001) were obtained from the Developmental Therapeutics Program repository (Rockville, MD); cisplatin was purchased from Enzo Life Sciences (Farmingdale, NY). DMSO was purchased from Sigma (St. Louis, MO). Cell Titer-Glo was purchased from Promega (Madison, WI). 96well solid tissue culture treated, black plates with clear bottoms were purchased from Corning Inc. (Corning, NY). PolyL-lysine was purchased from ScienCell Research Laboratories (ScienCell, Carlsbad, CA).

All work was conducted at the Frederick National Lab for Cancer Research in Frederick, Maryland.

Stock Solution and Treatment Medium Preparation and Use. BAT, NAB, and cisplatin were prepared as 200x stocks (of respective target concentrations) in DMSO. DMSO stocks were diluted directly into medium to obtain the final concentrations listed for the different models used. For CFU-GM, $10 \mu \mathrm{L}$ (or $20 \mu \mathrm{L}$ when a higher maximum concentration was chosen) of each respective BAT or NAB stock solution was added to $5 \mathrm{~mL}$ medium just before addition of $3.5 \mathrm{~mL}$ cell suspension. Immediately after, warmed $1.5 \mathrm{~mL}$ agarose solution was added to enable gelling in the culture plates. For 96well microplate studies using NRK or RBLAK cells complete medium was spiked with 200x stock solutions, spent medium was removed from all wells, and the freshly spiked medium was added on a daily basis. For rat liver slice studies, 200x stock solutions were spiked directly into vials containing enough medium for the respective treatment groups. The amount of medium spiked varied based on the number of slices being cultured for respective exposure lengths and number of slices in a group (typically 6-20 mL of medium was prepared per treatment/feeding). Stock solutions for the CFU-GM were prepared differently and are described in the CFU-GM assay section. NRK and RBLAK cells and PCLS had medium containing treatment (or just vehicle for controls) changed every day. The validated CFU-GM assay uses a semisolid agarose substrate that contains the medium and after plating the cells with their exposures, the medium cannot be changed.

\subsection{CFU-GM Assay}

Donor Bone Marrow. The CFU-GM clonogenic assay was conducted using freshly collected, human, dog (LonzaBiowhittaker, Walkersville, MD), or rat (Battelle, Columbus, $\mathrm{OH}$ ) bone marrow cells. All donors were healthy, prescreened individuals and the tissue samples were handled in accordance with the NIH Bloodborne Pathogens SOP for NCIFrederick, as well as in accordance with the institutional IBC guidelines. Bone marrow was collected from 6 donors on 3 different occasions. Bone marrow was processed and the purified mononuclear cells were shipped to our laboratory the following day.

Bone Marrow Cell Processing. Upon arrival, human or dog bone marrow cells were gently pelleted and the transport media removed. Rat bone marrow cells were isolated from rat femurs shipped on ice from Battelle as previously described [24] with the exception that no $\mathrm{NH}_{4} \mathrm{Cl}$ or Turks solution steps were performed. Cells from all species were then resuspended in $5 \mathrm{~mL}$ of Plasma-Lyte A USP (Baxter Healthcare, Deerfield, IL), mixed well and human cells were treated with $2.5 \mu \mathrm{L} / \mathrm{mL}$ Pulmozyme (Genentech, Inc., South San Francisco, CA). After a 10-minute room temperature incubation (human cells only), the cells were layered over $5 \mathrm{~mL}$ Ficol-Paque PLUS (Stem Cell Technologies, Vancouver, B.C.) and centrifuged for 30 minutes (without brakes) at 1500 relative centrifugal force to enrich the viable mononuclear cell population. The buffy layer containing the mononuclear cells (MNC's) was collected, washed in $14 \mathrm{~mL}$ Plasma-Lyte A USP, and finally resuspended in $10 \mathrm{~mL}$ IMDM (Stem Cell Technologies, Vancouver, B.C.). Cell counts were performed using a Beckman Coulter ViCell counter. 
Plating of Treated Cells for CFU-GM and IC90 Determination. Each treatment was prepared in separate $15 \mathrm{~mL}$ conical vials. A species-specific density of cell suspension $(3.5 \mathrm{~mL})$ was added to $5 \mathrm{~mL}$ medium containing 2000x stock drug for a final volume of $8.5 \mathrm{~mL}$ of complete medium and the vials placed in a $37^{\circ} \mathrm{C}$ water bath. After warming the agarose solution, tubes containing cells had drug stocks added $(2000 \mathrm{x}$ dilution for a volume of $10 \mathrm{~mL}$ ), and immediately after, $1.5 \mathrm{~mL}$ of 2.5\% SeaPlaque Agarose (Lonza-Biowhittaker, Walkersville, MD) in water was added to the cell/drug suspension, vortexed well, and $2 \mathrm{~mL}$ was plated in triplicate in 6-well plates containing a pregelled, $2 \mathrm{~mL}$ underlayer of IMDM, fetal bovine serum, and 2.5\% SeaPlaque Agarose per well. Each well of the 6-well plates contained 200,000 (human), 375,000-500,000 (dog), or 40,000 (rat) donor MMC. The plates were cooled at $4^{\circ} \mathrm{C}$ until completely gelled and then placed in an incubator at $37^{\circ} \mathrm{C}$ with $5 \% \mathrm{CO}_{2}$. After 14 days, the colonies ( $>64$ cells) were counted and IC90 values calculated (from the reduction in colonies per well), using the vehicle control as a reference.

2.3. NRK and RBLAK Cultures. NRK cells were obtained from the American Type Culture Collection (ATCC; Manassas, VA). RBLAK were obtained from CELLnTEC (distributed by Zen-Bio, Research Triangle Park, NC). NRK cells were cultured in Dulbecco's modified Eagle's medium containing $2 \mathrm{mM}$ glutamine, $1.5 \mathrm{~g} / \mathrm{L}$ sodium bicarbonate, and $4.5 \mathrm{~g}$ of glucose/L, $1 \mathrm{mM}$ sodium pyruvate (GIBCO-Invitrogen, Carlsbad, CA) supplemented with $5 \%$ heat inactivated fetal bovine serum (Lonza-Biowhittaker Walkersville, MD).

ATP Assay. Cells were seeded in 96-well plates with $100 \mu \mathrm{L}$ cell suspension $(40,000$ cells $/ \mathrm{mL})$ added per well for $24 \mathrm{hr}$ exposures. For $48 \mathrm{hr}, 72 \mathrm{hr}$, and $96 \mathrm{hr}$ exposures, $50 \mu \mathrm{L}$ cell suspension was added to wells already containing $50 \mathrm{~mL}$ medium. The lower cell seeding number was used to prevent overgrowth in the wells for the longer incubation times. Plated cells were maintained at $37^{\circ} \mathrm{C}$ in a humidified $5 \% \mathrm{CO}_{2} / 95 \%$ air atmosphere incubator. Total dilutions were either $500 \mathrm{x}$ or $1000 \mathrm{x}$ for a maximum vehicle concentration of $0.2 \%$. RBLAK were cultured in CnT-BM.16 basal medium supplemented with CnT 16 supplements obtained from CELLnTEC. The cell suspension was adjusted to 100,000 cells/mL and seeding volumes and culture conditions were the same as with NRK. After allowing cells to attach overnight, medium was removed and $100 \mu \mathrm{L}$ freshly spiked medium containing BAT, NAB, or cisplatin treatment was added to wells. After exposure completion, cells were lysed to measure relative ATP levels, determined in accordance with the manufacturer's protocol. In short, an equal volume of Cell Titer-Glo was added to the wells and the plate was shaken vigorously for $2 \mathrm{~min}$. Relative luminescence (RLU) was measured after $10 \mathrm{~min}$ on a TECAN plate reader (Research Triangle Park, NC). Vehicle control was used as a reference when percent of control values was determined.

$\gamma$-H2AX Assay. Cells were seeded in $100 \mu \mathrm{L}(100,000$ cells $/ \mathrm{mL})$ and cultured as above but for shorter time points. Following exposure, medium was removed and the cells were fixed and permeabilized for $5 \mathrm{~min}$. With $4 \%$ paraformaldehyde + $0.5 \%$ Triton-X in PBS, stained for $1 \mathrm{hr}$ at room temperature with an antibody specific to $\gamma$-H2AX (antiphosphohistone H2A.X [Ser139], clone JBW301, biotin conjugate, [Millipore]). Alexa fluor 594 conjugated streptavidin, (Alexa Fluor 594 [Invitrogen]) was added for $1 \mathrm{hr}$ at room temperature. Cells were stained with DAPI for $30 \mathrm{~min}$ to label the nucleus. Plates were imaged on a GE InCell 2000 Analyzer at the appropriate wavelengths, imaging 4 fields per well with a $20 \mathrm{x}$ objective in both channels. The resulting image stack was analyzed with the IN Cell Analyzer Workstation 3.7 using the Granularity Analysis Protocol. Total granule intensity/cell, total granule/cell, and total nuclei area were calculated using the analysis software. The percentage of the nucleus staining positive for $\gamma$-H2AX was calculated by dividing the total granule/cell by the total nuclei area.

2.4. PCLS Studies. All animal work was done in accordance with institutional and governmental guiding principles in the use and care of rats (the Frederick National Lab for Cancer Research is accredited by AAALAC International and follows the Public Health Service Policy for the Care and Use of Laboratory Animals). Animal care was provided in accordance with the procedures outlined in the "Guide for Care and Use of Laboratory Animals" [25].

Glutathione, bovine serum albumin (BSA), hydrocortisone 21-acetate, retinyl acetate, Triton X-100, and dimethyl sulfoxide (DMSO) were obtained from Sigma (St. Louis, $\mathrm{MO}$ ); Humulin N (Human insulin, rDNA origin) was obtained from Eli Lily (Indianapolis, IN); Hepatocyte Culture Media Kit (Cat\# 355056, BD Biosciences, Franklin Lakes, New Jersey), $\mathrm{Ca}^{+}$- and $\mathrm{Mg}^{+}$-free PBS, and antibiotic-antimycotic solution were obtained from Invitrogen/Gibco (Carlsbad, California); Viaspan (Belzer-University of Wisconsin [UW] cold storage solution) supplemented with glutathione (3 mM) was obtained from Fisher Scientific (Pittsburgh, Pennsylvania). Insulin/transferrin/selenium (ITS) was purchased from Sigma (cat\# I1884).

Equipment included the following: tissue-coring press and titanium inserts (Vitron; Tucson, Arizona); $0.45 \mu \mathrm{m}$ surfactant-free hydroanalysis mixed cellulose ester Tritonfree (HATF) filter paper used to support the PCLS slices in culture (Millipore; Bedford, Massachusetts); polytetrafluoroethylene (PTFE) membrane TF-200 and $0.2 \mu \mathrm{m}$ filters (Pall Life Sciences; Ann Arbor, MI); low-background glass scintillation vials (Research Products International; Mt. Prospect, Illinois); and TC-8 roller drum unit (New Brunswick Scientific; Edison, New Jersey).

PCLS Preparation. Rats were anesthetized with isoflurane inhalation. The liver perfused with cold UW solution removed and placed in a beaker of ice-cold UW. The lobes were separated with a scalpel and cored into cylinders of $8 \mathrm{~mm}$ diameter. The cores were then sliced in a cold, supplemented UW solution into $\sim 230 \mu \mathrm{m}$-thick discs using a Krumdieck slicer (Alabama Research and Development; Munford, Alabama). Uniformly shaped slices were selected for experiments. Using a sterile cotton swab, the slices were placed on sterile HATF paper inside the titanium inserts. All 
slice manipulations were done in ice-cold-supplemented UW solution.

Slice Equilibration/Culture. The titanium inserts with the slices were placed in sterile scintillation vials, each containing a $1.7 \mathrm{~mL}$ culture medium consisting of $\mathrm{BD}$ hepatocyte medium. The vials were then capped with sterilized open-end caps containing PTFE membrane filters, each held in place by a hole-punched Teflon liner to allow gas exchange with the external atmosphere. Vials were placed in the roller drum inside a humidified incubator at $37^{\circ} \mathrm{C}$ under a $75 \% \mathrm{O}_{2}, 5 \%$ $\mathrm{CO}_{2}$, and $20 \%$ air atmosphere. The roller drum containing the vials was rotated at $3 \mathrm{rpm}$. Following equilibration for $2-3 \mathrm{~h}$, the zero time point group was harvested. In a biosafety hood, the medium in the remaining vials was aspirated and replaced with the designated prewarmed media with or without $\mathrm{BAT}$ or NAB at designated concentrations. Control groups were exposed to DMSO at the same final concentration $(0.5 \% \mathrm{v} / \mathrm{v}$ DMSO $)$. Each treatment group was comprised of multiple replicates (slices cultured in separate tubes) and the slice medium in each vial was replaced with fresh medium daily until the slices were harvested. For treatment groups designated for BrdU staining, a final concentration of $10 \mu \mathrm{M}$ BrdU was added to slices $18-24 \mathrm{hr}$ prior to harvest.

Harvest and Biomarker Analysis. At the indicated time points, medium and slice samples were collected for processing and analysis. The collected medium $(1 \mathrm{~mL} / \mathrm{vial})$ was pooled for respective slices and stored at $4^{\circ} \mathrm{C}$ for subsequent biochemical analysis ( $<1$ week total storage). Slices and filter paper were removed from their titanium inserts and rinsed in PBS (room temp) by briefly submerging the slice (still on its HATF paper) into a vessel containing PBS. Each slice section designated for analysis was transferred into a $1.5 \mathrm{~mL}$ Eppendorf tube containing $0.5 \mathrm{~mL}$ PBS $+0.5 \%$ Triton X100 on ice. The sections were then homogenized and briefly sonicated at ice-cold temperatures. The resulting lysates were centrifuged at $9000 \times \mathrm{g}$ for $5 \mathrm{~min}$ to remove particulate matter. The resulting supernatants were stored at $4^{\circ} \mathrm{C}$ until the lysates and medium samples were analyzed. To examine the biomarker content, medium and lysate samples were sent on ice with the corresponding media (for baseline values) to the Pathology/Histotechnology Laboratory (PHL) for analysis of biomarker (AST, ALT, GGT, ALP for lysates only, and LDH) content. A chemiluminescent ALP-specific substrate purchased from Michigan Diagnostics (Royal Oak, MI) was used to measure ALP activity in the medium. This was done by extrapolating activity in the control and treatment groups from an ALP standard curve. All medium ALP assays were performed in 96-well microtiter plates. Lysate protein content was measured using the Pierce BCA protein assay kit (VWR International, West Chester, PA) and BSA standards in PBS + $0.5 \%$ Triton $\mathrm{X}-100$.

Harvest and PCLS Histology Preparation. The faces of slice sections were covered by lens paper (wetted in $10 \%$ zincbuffered formalin) and placed between two foam inserts. This "sandwich" was first placed in a histological cassette, fixed in $10 \%$ buffered formalin for $18-24 \mathrm{~h}$, then transferred to $70 \%$ alcohol, and submitted to the NCI-Frederick Pathology/Histotechnology Laboratory. Hematoxylin-eosin (H\&E) and PAS staining, BrdU, and $\gamma$-H2AX immunohistochemistry staining were done on $4 \mu \mathrm{m}$ thick tissue sections cut from the paraffin blocks. After digital imaging/scanning (using Aperio Slide Scanner) of slices for $\gamma$-H2AX the same sections were then $\mathrm{H} \& \mathrm{E}$ stained to enable direct comparison of stained regions.

Semiquantitative Histological Analysis. All H\&E and BrdU slice sections were assessed in a blind manner by a histopathologist. H\&E-stained slice sections were thoroughly examined under low (25x and 50x) and medium (100x and $200 \times$ ) magnifications and were assessed for viability (percent) and glycogen store (0-4) buildup. A viability percentage score was assigned to hepatocytes and biliary cells. PAS stained slides were used to confirm histological findings of glycogen buildup. Both $\mathrm{H} \& \mathrm{E}$ and BrdU stained sections were closely examined to score (0-4) slices for the level of biliary proliferation evident.

PCLS $\gamma$-H2AX Staining. All staining was performed by the PHL lab. The Leica Biosystems Bond-Max autostainer was used to stain slide sections that were first deparaffinized and antigen retrieved (using citrate solution included as one of the Bond Stainer reagents). A 1:100 dilution (60 min, RT) of primary antibody (antiphosphohistone H2AX (S139), clone JBW301, Millipore cat\# 16-193) was used to incubate with the slice sections. The $30 \mathrm{~min}$ secondary antibody was done using a Streptavidin, Alexa 594 conjugate (cat\# S11227) at $1: 100$ dilution in a Bond Wash solution (also ordered separate for the Bond autostainer and prepared as a 1:10 in $\mathrm{dH} 2 \mathrm{O}$ ). After rinsing DAPI (Invitrogen, cat\# D21490) was applied for $20 \mathrm{~min}$. (RT) as a 1:5000 dilution in the same Bond Wash as the secondary. Slides are then put in PBS and coverslipped using the mountant ProLong Gold antifade reagent made by Invitrogen (cat\# P3693). Slides were digitized using the Aperio Scanscope FL (Aperio, Vista, CA) slide scanner.

\subsection{Data Analysis}

CFU-GM. Control and treatment groups from all donors were run in triplicate and all evaluable groups had colony counts averaged and the vehicle control was used as a reference to generate $\%$ of control $(\% \mathrm{C})$ values. $\% \mathrm{C}$ values were averaged across replicate experiments conducted for all species. The resulting average $\% \mathrm{C}$ values generated a slope when loss of colony formation was observed and the values closest to $90 \%$ inhibition were used to calculate the IC90 concentration.

NRK and RBLAK: treatment groups replicate wells were averaged and for the ATP assay the vehicle control groups were used for \% of control calculation. Percent of control values was then averaged across replicate experiments and experimental variation was calculated as $\%$ coefficient of variation $(\% \mathrm{CV})$. For $\gamma-\mathrm{H} 2 \mathrm{AX}$ data presentation, averaged nuclear area positive (NAP) readings of treatment groups were compared to vehicle control. For statistical comparisons, 
homoscedastic Student's $t$-test was used to establish significant difference $(P<0.05)$.

PCLS. Replicate PCLS had data averaged within experiments and replicate experiment data was averaged across all three experiments. Variation of data was calculated as standard error of the mean (SEM) and when appropriate, values were calculated as \% of vehicle control. For purposes of statistical evaluation, biochemistry values were tested against respective controls within each separate experiment due to baseline variability of slice experiments. However, for histology assessments where general scores (0-4) were assigned to slices, data from the three experiments were pooled for treatment versus control group comparisons. If any slice treatment dataset consisted of less than 3 values (across all 3 experiments) over the detection threshold of the assay, the average data point was not included for analysis. The following day 7 treatment group medium $\mathrm{LDH}$ values were omitted due to this reason: vehicle control, $5 \mu \mathrm{M}$ BAT, $0.5 \mu \mathrm{M}$ NAB, and $2.5 \mu \mathrm{M}$ NAB. When significant difference was assessed between control and treatment groups, the datasets were compared using a single-tailed, homoscedastic Student's $t$-test, except for PCLS ALP, GGT, and AST content where a two-tailed test was used due to the potential for biphasic data, relative to control. For $\gamma$-H2AX evaluation NAP percentage was performed using Definiens Developer XD 2.0.3. A rule set was developed to segment nuclei based on the DAPI stain and then determine if an entire nucleus was either positive or negative for $\gamma$-H2AX using an intensity threshold. The total area of the positive nuclei was divided by the total area of all the nuclei to obtain percentages. Results for each exposure are from at least 3 slices in total, from at least 2 independent experiments. Slices were not evaluated if the software took longer than 1 hour to evaluate or if the slice did not mount to the slide properly.

\section{Results}

3.1. CFU-GM. In 3 separate experimental runs, Ficol MMC isolates from a total of 6 humans, 5 of 6 dog donors, and 3 sets of pooled cells from male F344 rat femurs for CFU-GM experimentation had viable colony formation during culture. While BAT, tested up to $50 \mu \mathrm{M}$, showed clear inhibition of colony formation and similar IC90 values across all 3 species (Table 1), NAB did not cause inhibition of colony formation. Due to solubility issues (compound precipitation) data from the highest concentrations of BAT and NAB used $(100 \mu \mathrm{M}$ and $10 \mu \mathrm{M})$, respectively, were not evaluated.

3.2. NRK and RBLAK ATP Assay. NRK and RBLAK cells were exposed to BAT, NAB, and cisplatin (as a positive control) in several replicate experiments, where BAT and cisplatin had target concentrations up to $100 \mu \mathrm{M}$ and NAB up to $10 \mu \mathrm{M}$. Both NRK and RBLAK cells were sensitive to BAT and cisplatin exposure in a time- and concentrationdependent manner (Figure 1). However, RBLAK cells were found to be more sensitive to BAT than NRK cells, with the greatest differences seen at $24 \mathrm{hr}$ and $48 \mathrm{hr}$ exposure lengths. Comparing the level of BAT-induced inhibition at concentrations found to be significantly lower $(P<0.05)$
TABLE 1: CFU-GM IC 90 results $(\mu \mathrm{M})$ following 14-day BAT exposure.

\begin{tabular}{|c|c|c|c|}
\hline Experiment & Rat & Dog & Human \\
\hline \multirow{2}{*}{$\# 1$} & \multirow{2}{*}{30} & 21 & 26 \\
\hline & & 21 & 27 \\
\hline \multirow{2}{*}{$\# 2$} & \multirow{2}{*}{25} & 23 & 28 \\
\hline & & $\mathrm{Na}$ & 27 \\
\hline \multirow{2}{*}{$\# 3$} & \multirow{2}{*}{32} & 17 & 30 \\
\hline & & 26 & 30 \\
\hline Average & 29.0 & 21.4 & 27.9 \\
\hline St. Dev. & 3.7 & 3.2 & 1.4 \\
\hline
\end{tabular}

Na: not available, poor growth in plates.

TABLE 2: Comparison of ATP assay $\mathrm{IC}_{50}$ values $(\mu \mathrm{M})^{*}$.

\begin{tabular}{lcccc}
\hline Cell & $24 \mathrm{hr}$ & $48 \mathrm{hr}$ & $72 \mathrm{hr}$ & $96 \mathrm{hr}$ \\
\hline NRK & 60 & 35 & 18 & 16 \\
RBLAK & 31 & 21 & 19 & 17 \\
\hline
\end{tabular}

${ }^{*}$ Calculations were made using averaged BAT data $(n=3-4$ experiments, 3 4 replicate wells/experiment).

than respective cell type controls, significantly more toxicity was seen in RBLAK at $24 \mathrm{hr}(25 \mu \mathrm{M}$ and $50 \mu \mathrm{M}), 48 \mathrm{hr}$ $(12.5 \mu \mathrm{M}, 25 \mu \mathrm{M}$, and $50 \mu \mathrm{M})$, and also at $72 \mathrm{hr}(50 \mu \mathrm{M})$. Calculation of IC50 values determined that RBLAK was approximately half those for NRK, but only at 24, and $48 \mathrm{hr}$ exposures (Table 2). At 72 and 96 hr exposures, no difference was detected between IC50 values for both of the cell types.

3.3. PCLS BAT and NAB Exposures. Slices maintained an optimal hepatic architecture and viability of approximately $70 \%$ and $90 \%$, for hepatocytes (HPC) and biliary epithelial cells (BEC), respectively, throughout the length of the 7 day studies (Figure 2). Biochemically, slices exhibited characteristics of biomarker retention over the culture period (Table 3 ) that were as good as or better (depending on the marker assessed) than previously reported [20, 21].

PCLS were exposed to concentrations of BAT and NAB that had no observed solubility issues, but where tissue effects may be observed based on previous studies and literature. BAT $(5 \mu \mathrm{M}$ and $25 \mu \mathrm{M})$ and NAB $(0.5 \mu \mathrm{M}$ and $2.5 \mu \mathrm{M})$ were exposed (with daily replacement of treatment medium) to PCLS and medium and tissue harvested for evaluation on days 1, 3, and 7 following treatment initiation. Leakage of $\mathrm{LDH}, \mathrm{AST}$, and ALP was quantified and while NAB did not affect $\mathrm{LDH}, \mathrm{AST}$, or ALP release, BAT did appear to elevate $\mathrm{LDH}$ levels in the medium at days 3 and 7 (25 $\mu \mathrm{M}$ only) after exposure initiation (Figure 3 ). Concomitant elevation of AST at the same time points indicates an impact on HPC viability. Exposure of NAB resulted in a more modest, but significant increase of AST at the day 3 time point (at both $0.5 \mu \mathrm{M}$ and $2.5 \mu \mathrm{M}$ ). Despite the slight increase in medium $\mathrm{LDH}$ at days 1 and $3,(2.5 \mu \mathrm{M}$ only $)$ was not significantly higher than control in any of the 3 replicate experiments. The BEC marker ALP, also measured in the medium, demonstrated 

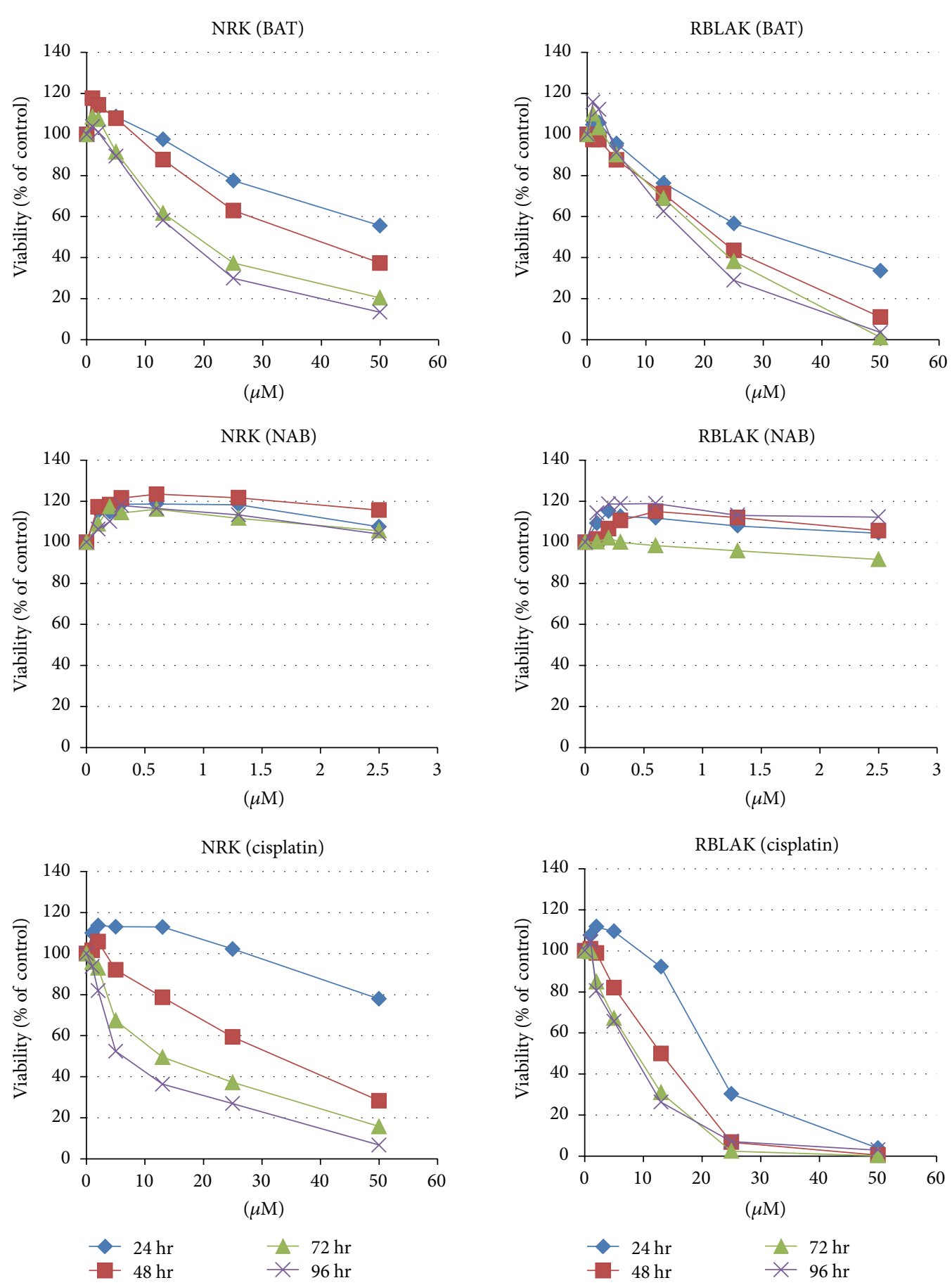

FIGURE 1: Batracylin, but not N-acetyl batracylin, induces loss of NRK kidney cell and RBLAK bladder cell viability at soluble concentrations. Both cell types are sensitive to cisplatin, the positive control used as the reference compound for kidney and bladder toxicity (bottom row).

significant change after both BAT and NAB exposure (Figure 3 ), suggesting both compounds impacted these cells.

Evaluation of PCLS tissues revealed trends consistent with those of released markers. The general markers, PCLS protein, and $\mathrm{LDH}$ content reveal that both $\mathrm{BAT}$ and $\mathrm{NAB}$ (to a lesser extent) caused PCLS LDH content to diminish, primarily at days 3 and 7 (Table 3). Assessment of tissue lysate for the HPC markers AST and ALT and with histological examination of PCLS revealed that both BAT and NAB impacted HPC viability and biomarker content (Table 3 ). Exposure to $25 \mu \mathrm{M}$ BAT eliminated glycogen buildup in HPC and elicited the most severe changes in HPC marker content. PCLS AST content was elevated while ALT levels diminished as was previously noted during compound induced liver slice toxicity [19-21]. While NAB exposure resulted in a significant HPC viability drop at day 3 , exposure to $25 \mu \mathrm{M}$ BAT resulted 


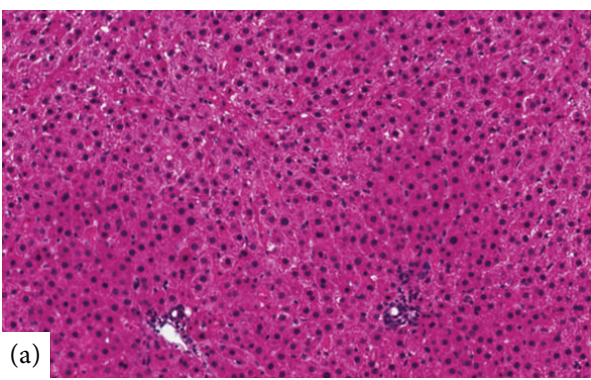

(a)

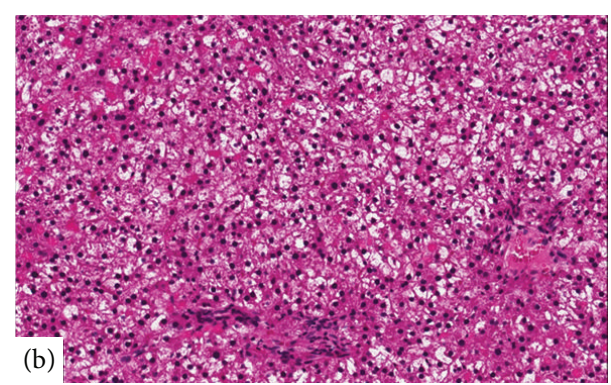

(b)

FIGURE 2: Control PCLS on (a) the day of slicing and (b) 7 days after culture. A high degree of cell viability is retained and buildup of glycogen stores is evident. H\&E 200x.

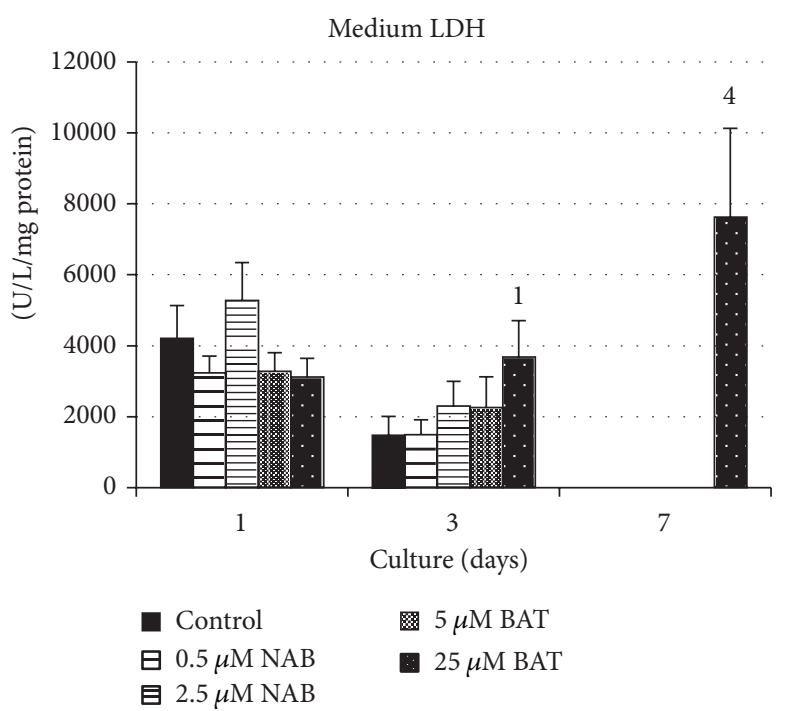

(a)

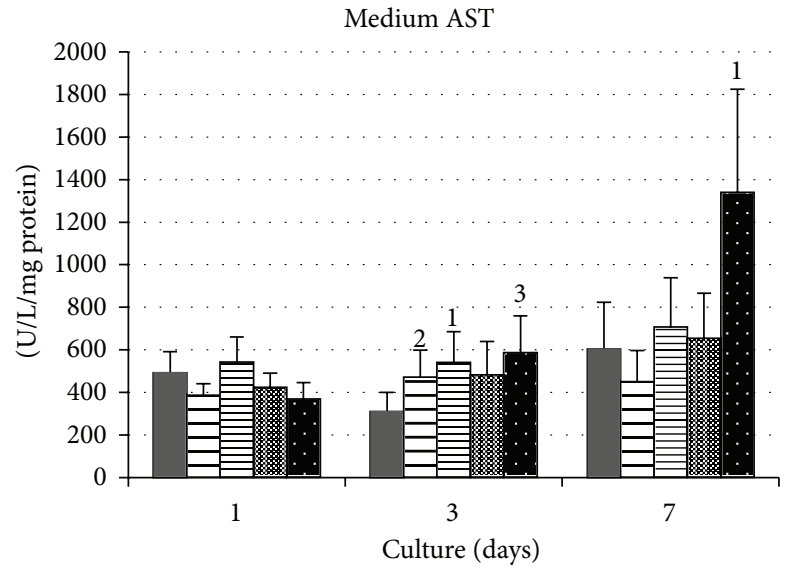

$\square$ Control
$\square 0.5 \mu \mathrm{M}$ NAB
目 $2.5 \mu \mathrm{M}$ NAB

(b)

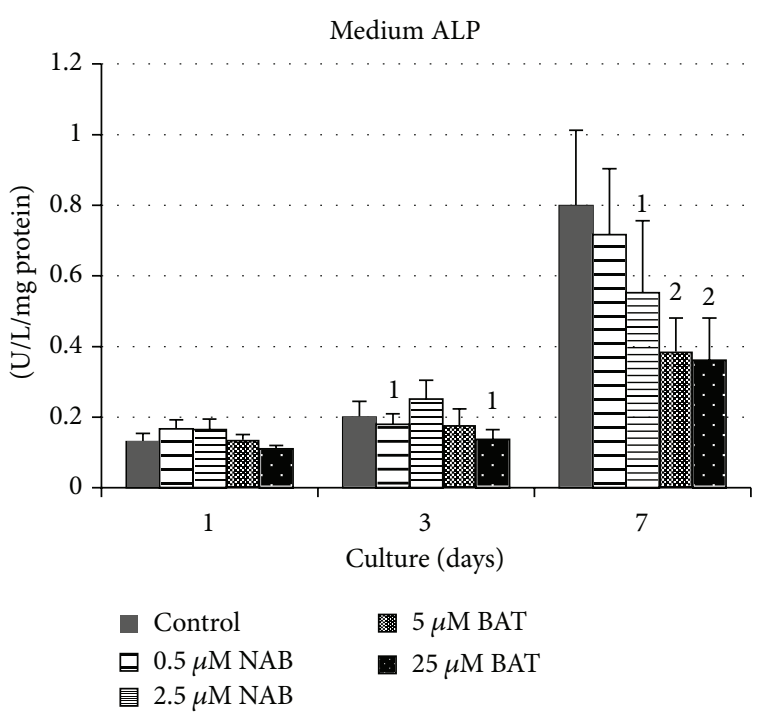

(c)

FIGURE 3: Biomarkers in medium following BAT or NAB exposure. BAT exposure elicits elevated levels of LDH at days 3 and 7. On day 7, $5 \mu$ M BAT and $0.5 \mu \mathrm{M}$ and $2.5 \mu \mathrm{M}$ NAB groups all had $<3$ values measurable in medium. Data corresponding to these groups was not graphed. Due to low sampling size significance was not calculated for ALP on day $1 .{ }^{1}$ one, ${ }^{2}$ two, or ${ }^{3}$ three experiments (of three total) demonstrated significantly greater (AST, LDH) or lower (ALP) marker content in the medium versus control medium values. $P<0.05 .{ }^{4}$ significance not be calculated since $<3$ marker values were measurable in the control medium. 


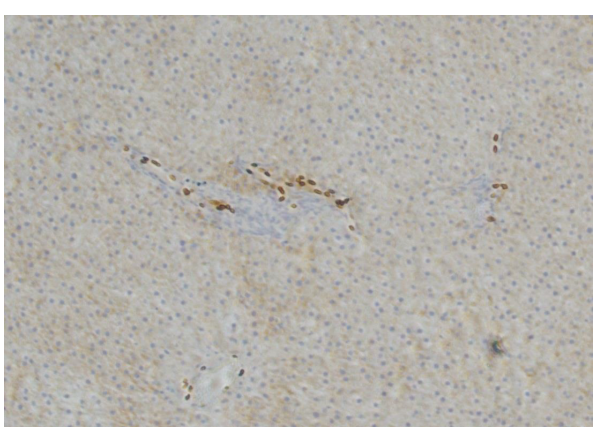

(a)

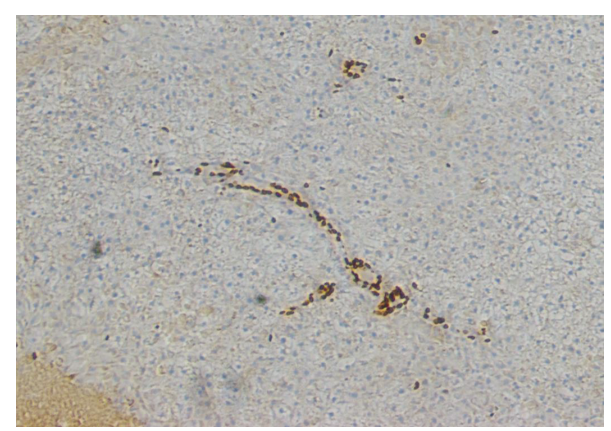

(b)

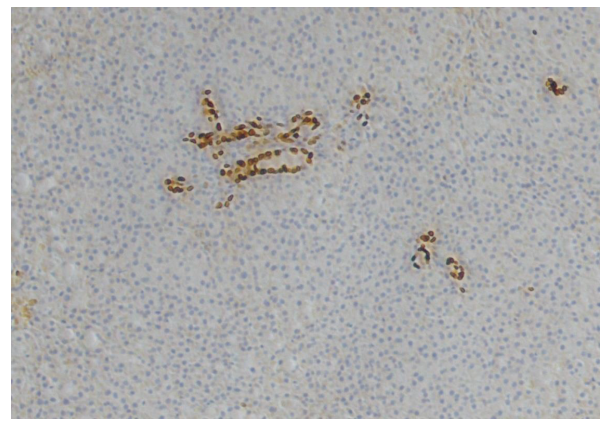

(c)

Figure 4: Increased BrdU positive BEC nuclei following BAT or NAB exposure. At day 7, (a) Control PCLS exhibit significantly less BrdU positive BEC nuclei than PCLS exposed to (b) $2.5 \mu \mathrm{M}$ NAB or (c) $25 \mu \mathrm{M}$ BAT. BrdU stain, 10x objective.

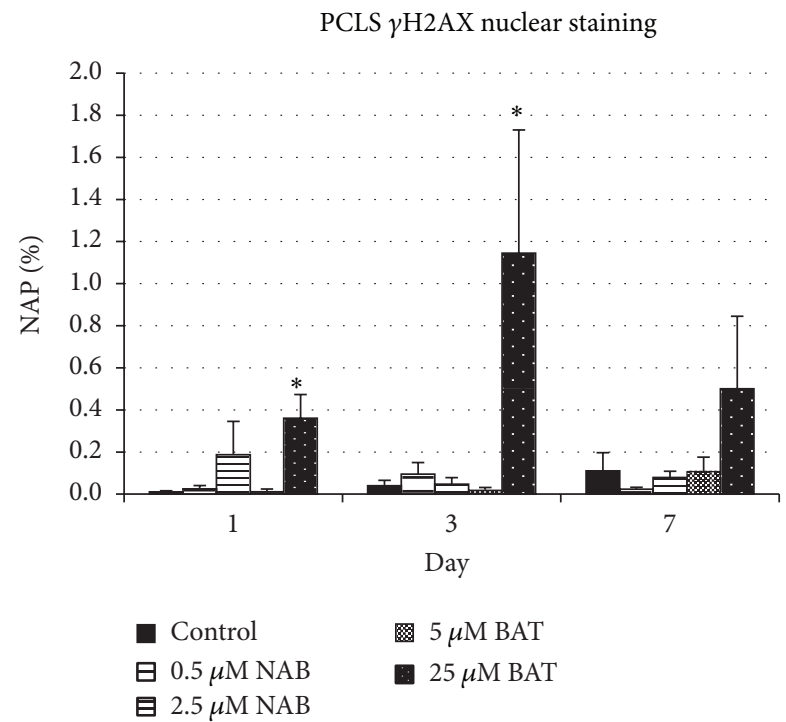

FIGURE 5: Increase in \%NAP of slices exposed to BAT. While elevations of staining are noted with $2.5 \mu \mathrm{M} \mathrm{NAB}$ at day 1 , significant increases versus vehicle control (VC) were measured in slices exposed to $25 \mu \mathrm{M}$ BAT. By day 7, damage is substantial to the $25 \mu \mathrm{M}$ BAT treated PCLS and \%NAP has subsided.

in a drop to $51 \pm 4 \%$ by day 3 and caused a further drop to $41 \pm 4 \%$ by day 7 . Interestingly, the effects of NAB are most prominent at day 3 , but differences versus control subside by the day 7 time point. The extent of $25 \mu \mathrm{M}$ BAT-induced damage is also reflected by the loss of PCLS protein content by day 7 ( $59 \pm 9 \%$ of control).

Both BAT and NAB exposures affected the BEC in PCLS at all concentrations tested (Table 3). While histological evaluation of stained PCLS sections indicated no significant loss of viability with either BAT or NAB exposure, bile duct proliferation was noted on the H\&E-stained sections of slices exposed to either compound. These histological findings were confirmed using BrdU staining (Figure 4). While the BEC viability was not affected by either molecule (histological observations, data not shown), both molecules caused a drop in ALP and GGT levels in the tissue slices, as well as in the medium.

3.4. $\gamma$-H2AX Assay. Exposure of NRK and RBLAK cells to BAT or NAB resulted in increases in the \%NAP for $\gamma$-H2AX staining (Table 4). Exposure of NRK cells to BAT $(50 \mu \mathrm{M})$ resulted in a maximal increase of \%NAP of approximately 10 -fold over control values at $6 \mathrm{hr}$ but dimished to a $\sim 7$-fold increase by $72 \mathrm{hr}$. RBLAK were found to be more sensitive to lower concentrations of BAT, with a 5 -fold increase of $\% \mathrm{NAP}$ over control at $3.1 \mu \mathrm{M}(6 \mathrm{hr})$, a slightly lesser fold maximum over control ( $\sim$-fold at $50 \mu \mathrm{M}$ BAT at $6 \mathrm{hr})$, but also a diminished increase by $72 \mathrm{hr}$. Although NAB was applied at lower concentrations, significant increases of \%NAP over control were also seen. Increases of $\% \mathrm{NAP}$ up to $\sim 2$-fold of control were seen for NRK and up to 2.5 -fold for RBLAK, and these increases were both observed at $72 \mathrm{hr}$.

Quantitation of PCLS sections stained for $\gamma$-H2AX revealed that $25 \mu \mathrm{M}$ BAT caused increases of \%NAP relative 
TABLE 3: PCLS lysate marker content changes with treatment.

\begin{tabular}{|c|c|c|c|c|c|c|c|c|c|c|c|}
\hline \multirow{2}{*}{ Group } & \multirow{2}{*}{ Day } & $\mathrm{LDH}$ & $\mathrm{LP}$ & GGT & AST & ALT & Protein & HPC & BEC & BEC prolif. & Gly \\
\hline & & \multicolumn{5}{|c|}{$\mathrm{U} / \mathrm{L} / \mathrm{mg}$ protein } & $\mathrm{mg}$ & \multicolumn{2}{|c|}{$\%$ Viable } & \multicolumn{2}{|c|}{$0-4$} \\
\hline \multirow{3}{*}{ Vehicle control } & 1 & $35082 \pm 4604$ & $69 \pm 12$ & $8 \pm 1$ & $4424 \pm 273$ & $812 \pm 79$ & $1.1 \pm 0.1$ & $72 \pm 3$ & $90 \pm 1$ & $0.0 \pm 0.0$ & 0.1 \\
\hline & 3 & $36448 \pm 4924$ & $116 \pm 10$ & $22 \pm 3$ & $4015 \pm$ & $562 \pm 77$ & $0.9 \pm 0.1$ & $71 \pm 3$ & $88 \pm 1$ & $0.0 \pm 0.0$ & $0.7 \pm 0.1$ \\
\hline & 7 & $30414 \pm 4330$ & $231 \pm 45$ & $153 \pm 22$ & $3710 \pm 357$ & $741 \pm 149$ & $0.7 \pm 0.1$ & $70 \pm 3$ & $93 \pm 1$ & & $1.9 \pm 0.2$ \\
\hline \multirow{3}{*}{$0.5 \mu \mathrm{M}$ NAB } & 1 & $30446 \pm 3961$ & $60 \pm 8$ & $7 \pm 2$ & $4282 \pm$ & $636 \pm 88$ & $1.1 \pm 0.2$ & $72 \pm 4$ & $87 \pm 2$ & $0.0 \pm 0.0$ & \pm 0.1 \\
\hline & 3 & $32860 \pm 4669$ & $149 \pm 28$ & $18 \pm 3$ & $4486 \pm$ & $555 \pm 84$ & $0.9 \pm 0.1$ & $60 \pm 3$ & $85 \pm 1$ & 0.1 & \pm 0.2 \\
\hline & 7 & $78 \pm 4$ & $210 \pm 46$ & $120 \pm 18$ & $3430 \pm$ & & & 3 & $91 \pm 1$ & & 0.3 \\
\hline \multirow{3}{*}{$2.5 \mu \mathrm{M}$ NAB } & 1 & $0 \pm$ & $82 \pm$ & $7 \pm 1$ & & & & \pm 5 & $84 \pm 4$ & & \\
\hline & 3 & $40 \pm 5243$ & $128 \pm 24$ & $18 \pm 3$ & $4867 \pm 663$ & $532 \pm 79$ & .1 & $51 \pm 5$ & $80 \pm 4$ & 0.0 & $=0.1$ \\
\hline & 7 & $996 \pm 4083$ & $155 \pm 28$ & $83 \pm 15$ & $4031 \pm 555$ & $607 \pm 154$ & $0.7 \pm 0.1$ & $62 \pm 4$ & $90 \pm 1$ & $1.8 \pm 0.3$ & $1.7 \pm 0.2$ \\
\hline \multirow{3}{*}{$5 \mu \mathrm{M}$ BAT } & 1 & $34629 \pm 4768$ & $66 \pm 9$ & $9 \pm 1$ & $4167 \pm 255$ & $777 \pm 63$ & $1.1 \pm 0.1$ & $77 \pm 3$ & $90 \pm 0$ & $0.0 \pm 0.0$ & $0.1 \pm 0.1$ \\
\hline & 3 & $35693 \pm 6354$ & $103 \pm 17$ & $22 \pm 5$ & $4507 \pm 534$ & $574 \pm 127$ & $0.8 \pm 0.1$ & $59 \pm 4$ & $85 \pm 2$ & $=0.1$ & $0.4 \pm 0.2$ \\
\hline & 7 & $26113 \pm 3956$ & $148 \pm 28$ & $94 \pm 18$ & $4130 \pm 576$ & $601 \pm 149$ & $0.6 \pm 0.1$ & $60 \pm 4$ & $86 \pm 3$ & $1.4 \pm 0.2$ & $1.4 \pm 0.2$ \\
\hline \multirow{3}{*}{$25 \mu \mathrm{M}$ BAT } & 1 & $33832 \pm 4577$ & $55 \pm 11$ & $6 \pm 1$ & $3920 \pm 304$ & $948 \pm 283$ & $1.3 \pm 0.2$ & $70 \pm 4$ & $87 \pm 1$ & $0.0 \pm 0.0$ & $0.0 \pm 0.0$ \\
\hline & 3 & $31164 \pm 5851$ & $68 \pm 10$ & $18 \pm 3$ & $5664 \pm 550$ & $461 \pm 53$ & $0.6 \pm 0.1$ & $51 \pm 4$ & $83 \pm 2$ & $0.1 \pm 0.1$ & $0.0 \pm 0.0$ \\
\hline & 7 & $22341 \pm 3608$ & $94 \pm 12$ & $110 \pm 21$ & $6919 \pm 1022$ & $370 \pm 47$ & $0.4 \pm 0.0$ & $41 \pm 4$ & $88 \pm 1$ & $1.4 \pm 0.3$ & $0.0 \pm 0.0$ \\
\hline
\end{tabular}

Bold fields indicate one or more experiments demonstrated a significant difference from vehicle control. BEC proliferation and glycogen content were assigned scores $(0-4)$ by a pathologist and averages were not assessed statistically.

TABLE 4: \% nuclei area positive for $\gamma$-H2AX staining.

\begin{tabular}{|c|c|c|c|c|c|c|c|}
\hline \multirow{2}{*}{ Treatment } & \multirow{2}{*}{$\mu \mathrm{M}$} & \multicolumn{3}{|c|}{ NRK } & \multicolumn{3}{|c|}{ RBLAK } \\
\hline & & $6 \mathrm{hr}$ & $24 \mathrm{hr}$ & $72 \mathrm{hr}$ & $6 \mathrm{hr}$ & $24 \mathrm{hr}$ & $72 \mathrm{hr}$ \\
\hline Vehicle & 0 & $3.3 \pm 0.6$ & $3.0 \pm 0.7$ & $1.3 \pm 0.1$ & $1.9 \pm 0.2$ & $2.0 \pm 0.3$ & $2.9 \pm 0.6$ \\
\hline \multirow{5}{*}{ BAT } & 3.13 & $4.8 \pm 1.0$ & $5.2 \pm 0.2$ & $2.6 \pm 0.5$ & $9.5 \pm 1.5$ & $12.2 \pm 0.3$ & $7.1 \pm 1.9$ \\
\hline & 6.25 & $5.5 \pm 0.7$ & $6.2 \pm 0.7$ & $3.4 \pm 0.6$ & $8.0 \pm 1.4$ & $10.3 \pm 0.5$ & $6.3 \pm 1.5$ \\
\hline & 12.5 & $7.9 \pm 0.8$ & $8.9 \pm 1.0$ & $5.1 \pm 0.5$ & $9.1 \pm 1.3$ & $8.8 \pm 0.8$ & $6.3 \pm 1.6$ \\
\hline & 25 & $19.0 \pm 2.6$ & $15.9 \pm 1.7$ & $6.7 \pm 0.8$ & $10.9 \pm 1.8$ & $5.2 \pm 0.4$ & $7.3 \pm 0.7$ \\
\hline & 50 & $30.9 \pm 3.3$ & $26.7 \pm 2.2$ & $8.9 \pm 2.1$ & $15.3 \pm 1.9$ & $12.1 \pm 0.2$ & $1.5 \pm 0.4$ \\
\hline \multirow{5}{*}{ NAB } & 0.31 & $3.6 \pm 0.3$ & $2.6 \pm 0.5$ & $1.6 \pm 0.2$ & $1.5 \pm 0.3$ & $1.9 \pm 0.1$ & $3.4 \pm 0.8$ \\
\hline & 0.63 & $3.4 \pm 0.6$ & $2.9 \pm 0.3$ & $1.4 \pm 0.2$ & $1.8 \pm 0.5$ & $2.3 \pm 0.2$ & $4.5 \pm 2.1$ \\
\hline & 1.25 & $3.7 \pm 0.7$ & $3.2 \pm 1.4$ & $1.8 \pm 0.4$ & $2.8 \pm 0.6$ & $2.1 \pm 0.6$ & $4.3 \pm 0.9$ \\
\hline & 2.5 & $3.7 \pm 0.2$ & $4.4 \pm 2.3$ & $2.1 \pm 0.3$ & $3.2 \pm 0.2$ & $2.9 \pm 0.6$ & $4.7 \pm 0.9$ \\
\hline & 5 & $4.3 \pm 0.3$ & $3.4 \pm 1.0$ & $2.5 \pm 0.4$ & $4.8 \pm 1.2$ & $4.3 \pm 0.7$ & $7.2 \pm 2.3$ \\
\hline \multirow{2}{*}{ Etoposide } & 3.13 & $40.7 \pm 1.2$ & $17.9 \pm 1.4$ & $13.2 \pm 1.5$ & $40.7 \pm 2.2$ & $40.9 \pm 0.6$ & $34.8 \pm 9.0$ \\
\hline & 25 & $74.6 \pm 4.6$ & $42.0 \pm 3.1$ & $27.3 \pm 2.8$ & $68.1 \pm 2.4$ & $35.5 \pm 10.2$ & $19.8 \pm 0.9$ \\
\hline
\end{tabular}

to control (Figure 5). Although $2.5 \mu \mathrm{M}$ NAB caused an increase at day 1 , it was not found significant. When comparing the merged dual channel photographs of the $25 \mu \mathrm{M}$ BAT groups at day 3 with the same slice section (subsequently stained for $\mathrm{H} \& \mathrm{E}$ ), a pattern of necrosis emerges. $\gamma$-H2AX positive nuclei appear to display greater $\gamma$-H2AX staining intensities closer to the interface of HPC necrosis and viable cells (Figure 6). Histologically, the prominent BAT-induced HPC toxicity appeared to depict a centrilobular/Zone 3 predilection.

\section{Discussion}

BAT was considered a potential chemotherapeutic when it demonstrated efficacy against adriamycin-resistant P388 leukemia, colon tumors [1], and subsequently other solid tumors [2]. Mechanistically, BAT was confirmed to inhibit Topo II [3] and these studies evaluated BAT and its analogs for efficacy against the HL-60 and potency for Topo II inhibition. Studies examining BAT efficacy (including the use of $\gamma$ $\mathrm{H} 2 \mathrm{AX}$ as a marker) and acetylation (with the use of human HPC) determined tumor growth inhibition and HPC toxicity occurs in the low $\mu \mathrm{M}$ range $[4,5,26]$. With data (in vitro and in vivo) indicating the biotransformation of BAT to NAB leads to greater toxicity, the level of toxicity exhibited in preclinical animal species tested reflects this (rats $>$ mice $\gg$ dogs) and that oral administration of NAB resulted in almost nil bioavailability [8-10] the question of how humans would fare after BAT exposure is perhaps dependent on the acetylation capability of the individual. Indeed human liver is 
Untreated control day 3
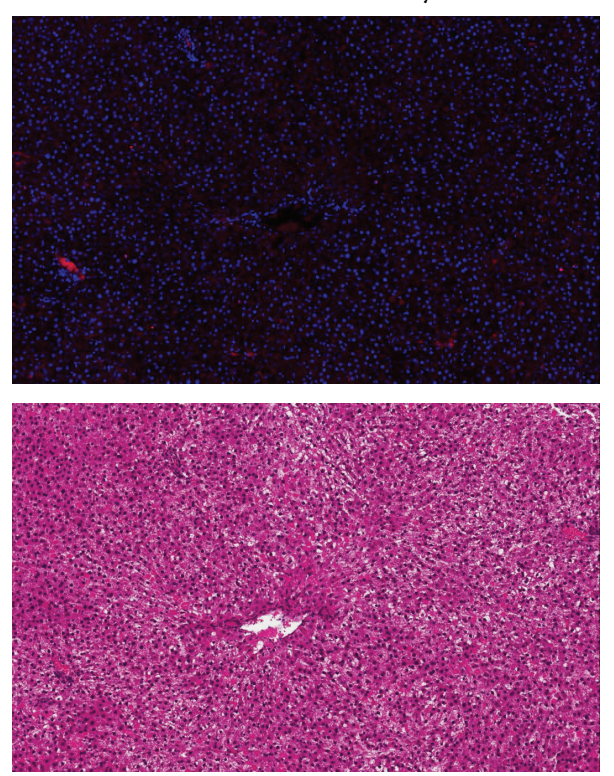

$25 \mu \mathrm{M}$ batracylin day 3
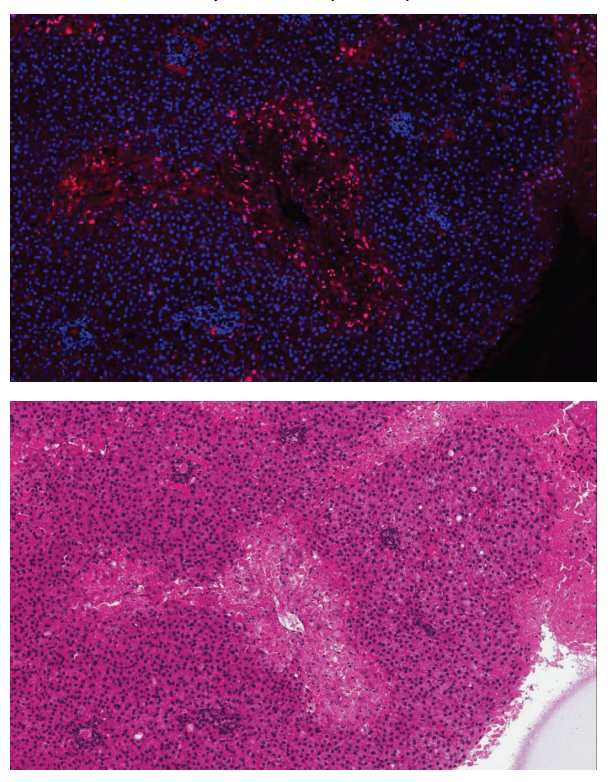

Figure 6: Increased $\gamma$-H2AX staining following $25 \mu \mathrm{M}$ batracylin exposure for 3 days (50x). All three replicate experiments demonstrated a large increase of $\gamma$-H2AX staining at this concentration, especially in centrilobular areas. By day 7 , the strong $\gamma$-H2AX staining has subsided and PCLS exposed to $25 \mu \mathrm{M}$ show substantial damage to HPC.

known to have two N-acetyl transferases, NAT 1 and NAT2, with BAT being the preferential substrate for the latter [9]. For this reason, and the human population consisting of rapid and nonrapid acetylators, the contribution of NAB to BAT exposure-induced adverse effects has been the subject of ongoing investigation.

Preclinical in vivo studies have determined that bone marrow, kidney, and liver were among the tissues adversely affected by in vivo BAT exposure. Clinical findings indicated bladder toxicities, initially not examined in vivo, were of concern (personal communication with the NCI). We chose cell and tissue models that were representative of these tissues and exposed them to both BAT and NAB in order to differentiate between cell-type specific and molecule-specific toxicities. As previously reported by multiple sources (and as determined in our laboratory), the solubility issue of NAB has imposed limits of exposures in the different model systems and prompted determining acceptable vehicle (DMSO) limits for the cultures so that maximal exposures could be conducted. Using $0.5 \%$ final DMSO in the media, BAT was found soluble to $\sim 50 \mu \mathrm{M}$ and $\mathrm{NAB}$ to $\sim 5 \mu \mathrm{M}$ in an aqueous medium, as determined by visual observation of precipitate formation. NRK, RBLAK, and rat PCLS were not adversely impacted by this DMSO concentration. In the CFU-GM assay, using hematopoietic progenitor cells often sensitive to the effects of higher concentrations of vehicle, only 1 donor in the NAB study demonstrated sensitivity to the vehicle control, but this did not impact the results as no NAB toxicity was evident. The BAT molecule was also found to have inherent fluorescence in the FITC channel so viability assays utilizing this channel (e.g., Calcein AM live cell esterase assay) were not found suitable for evaluating the toxicity.
BAT-induced myelosuppression was reported for preclinical studies using mouse, rat, and dog species [6]. Using rat, dog, and human bone marrow isolates our CFU-GM assay demonstrated that BAT exposure elicited a similar sensitivity ( $\mathrm{IC}_{90}$ range of $21-29 \mu \mathrm{M}$ ) across the species tested. With CYP3a (primarily found in the liver and intestine) having been identified as the enzyme responsible for acetylation of BAT and NAB having poor solubility, it is reasonable to conclude that concentrations of NAB that may cause hematopoietic toxicity are not achieved in the bone marrow. With dogs being poor acetylators, it can be assumed the bone marrow receives a BAT exposure devoid of breakdown. However, other fast acetylating species such as rat could potentially have higher concentrations of $\mathrm{NAB}$ exposing the bone marrow (assuming no solubility issues). The CFU-GM assay allowed us to deliver concentrations of BAT without metabolism or issues with precipitation. Therefore, it is assumed that the toxicity we detected across the species tested in the CFU-GM assay is due to BAT and not due to a breakdown or metabolic product. NAB, up to $5 \mu \mathrm{M}$, did not inhibit growth but the potential for NAB to cause colony growth inhibition at higher concentrations cannot be excluded.

Bladder and kidney damage has been linked to BAT exposure and our ATP assay results using NRK and RBLAK cells showed BAT-induced toxicities in both cell types. Bladder cells were more sensitive at the $24 \mathrm{hr}$ and $48 \mathrm{hr}$ time points, with IC50 values approximately half those of the NRK kidney cells (Table 2). However, RBLAK were also found more sensitive to the positive control (Cisplatin) in a similar manner. Again, NAB (up to $5 \mu \mathrm{M}$ ) showed no signs of toxicity to either cell type with exposures up to $96 \mathrm{hr}$. The $\gamma$-H2AX assay supported the ATP assay results by demonstrating a 
large difference in \%NAP increase between the two molecules (Table 4). While NAB did cause notable \%NAP increases at the highest concentration for the longer exposure period ( $96 \mathrm{hr}$ ), this was not sufficient to result in cell viability loss as determined in the ATP assay. While kidney damage resulting from BAT exposure is reported in preclinical studies, bladder damage was assessed during clinical studies and prompted its examination in preclinical models. Reported bladder damage to BAT exposed rats does with BAT $(32 \mathrm{mg} / \mathrm{kg}$ ) includes a small but insignificant decrease of bladder lumen wall and no increased $\gamma-\mathrm{H} 2 \mathrm{AX}$ staining (relative to control) at $24 \mathrm{hr}$ after dosing (personal communication with the NCI). Our observations suggest $>10 \mu \mathrm{M}$ BAT exposure is required to elicit a $\sim 20 \%$ loss of ATP in epithelial progenitor cells. Perhaps repeat or longer exposures to rats in vivo would lead to the detection of significant bladder toxicities.

The exposure of BAT and NAB to PCLS provided the opportunity to examine two adverse effects reported in rat liver: HPC toxicity and biliary hyperplasia. While NAB was largely ineffective in causing changes to HPC (as assessed biochemically, histologically, and with $\gamma$-H2AX staining) BAT caused severe damage to HPC following 7 days of exposure. However, when assessing BEC proliferation, both BAT and NAB caused substantial changes in BEC biochemical markers (medium and slices) and increases in the BEC proliferation scores. BrdU staining concurred that BEC were proliferating at a higher rate than control groups (Figure 4).

In summary, we have utilized in vitro and ex vivo models corresponding to cells or tissues known to be adversely affected by BAT exposure in vivo. While BAT exposure demonstrated toxicity in hematopoietic, kidney, and bladder cells, the lack of NAB toxicity may well be due to the inherent solubility limit of this molecule. The lack of bioavailability of orally administered NAB has also been noted in vivo and has hampered in vivo studies designed to evaluate its toxicity. The use of rat PCLS provided a unique model system that is metabolically capable of transforming BAT to NAB (a CYP3A mediated event) and therefore the conversion of BAT to its $\mathrm{NAB}$ metabolite could result in local concentrations higher than the solubility achieved in medium containing $0.5 \%$ DMSO vehicle. Exposure of PCLS to $25 \mu \mathrm{M}$ BAT resulted in substantial HPC destruction by day 7. The HPC marker changes observed with $2.5 \mu \mathrm{M}$ NAB exposure suggest that higher, local concentrations may be capable of doing so. However, the ability of NAB (at both 0.5 and $2.5 \mu \mathrm{M}$ ) to cause an increase in BEC proliferation was clearly evident as it was with BAT exposure. Using the PCLS model has allowed the identification of centrilobular/Zone 3 predilection of HPC damage and has implicated NAB in causing the biliary hyperplasia noted preclinically in rats.

\section{Conflict of Interests}

The authors declare that there is no conflict of interests regarding the publication of this paper.

\section{Acknowledgments}

The authors would like to thank Mike Furniss and John Hamre III for their technical expertise. The pathological evaluation of slice tissue was conducted by Dr. Khalid Amin, consultant for SAIC-Frederick. F344 rat liver tissue was obtained from Dr. Melinda Hollingshead and Suzie Borgel of the Biological Testing Branch at NCI-Frederick. Embedding, fixing, and staining the PCLS tissue were conducted by Tammy Beachly, Simona Florea, and Donna Butcher of the NCI-Frederick Pathology/Histotechnology Laboratory. This project has been funded in whole or in part with federal funds from the National Cancer Institute, National Institutes of Health, under Contract no. HHSN261200800001E. The content of this publication does not necessarily reflect the views or policies of the Department of Health and Human Services, nor does mention of trade names, commercial products, or organizations imply endorsement by the US Government. This research was supported [in part] by the Developmental Therapeutics Program in the Division of Cancer Treatment and Diagnosis of the National Cancer Institute.

\section{References}

[1] J. Plowman, K. D. Paull, G. Atassi et al., "Preclinical antitumor activity of batracylin (NSC 320846)," Investigational New Drugs, vol. 6, no. 3, pp. 147-153, 1988.

[2] P. Mucci-LoRusso, L. Polin, M. Bissery et al., "Activity of batracylin (NSC-320846) against solid tumors of mice," Investigational New Drugs, vol. 7, no. 4, pp. 295-306, 1989.

[3] Y. Luo, Y. Ren, T. Chou et al., "A structure-activity relationship study of batracylin analogues," Pharmaceutical Research, vol. 10, no. 6, pp. 918-923, 1993.

[4] S. K. Meegalla, G. J. Stevens, C. A. McQueen et al., "Synthesis and pharmacological evaluation of isoindolo[1,2-b]quinazolinone and isoindolo[2,1-a]benzimidazole derivatives related to the antitumor agent batracylin," Journal of Medicinal Chemistry, vol. 37, no. 20, pp. 3434-3439, 1994.

[5] V. A. Rao, K. Agama, S. Holbeck, and Y. Pommier, "Batracylin (NSC 320846), a dual inhibitor of DNA topoisomerases I and II induces histone $\gamma$-H2AX asa biomarker of DNA damage," Cancer Research, vol. 67, no. 20, pp. 9971-9979, 2007.

[6] M. El-hawari, M. L. Stoltz, and F. Pallas, Species Differences in the Toxic Response to Batracylin (NSC-320846), American Association for Cancer Research, San Francisco, Calif, USA, 2009.

[7] G. J. Stevens, J. L. Burkey, and C. A. McQueen, "Toxicity of the heterocyclic amine batracylin: investigation of rodent $\mathrm{N}$ acetyltransferase activity and potential contribution of cytochrome P450 3A," Cell Biology and Toxicology, vol. 16, no. 1, pp. 31-39, 2000.

[8] G. J. Stevens, E. J. Lavoie, and C. A. McQueen, "The role of acetylation in the mutagenicity of the antitumor agent, batracylin," Carcinogenesis, vol. 17, no. 1, pp. 115-119, 1996.

[9] G. J. Stevens, M. Payton, E. Sim, and C. A. Mcqueen, "N-acetylation of the heterocyclic amine batracylin by human liver," Drug Metabolism and Disposition, vol. 27, no. 9, pp. 966-971, 1999.

[10] M. M. Ames, D. A. Mathiesen, and J. M. Reid, "Differences in $\mathrm{N}$-acetylation of the experimental antitumor agent batracylin in the mouse and the rat," Investigational New Drugs, vol. 9, no. 3, pp. 219-225, 1991.

[11] S. G. Gómez, J. A. Bueren, G. Faircloth, and B. Albella, "Use of CFU-GM assay for prediction of human maximum tolerated dose of a new antitumoral drug: yondelis(ET-743)," Toxicology in Vitro, vol. 17, no. 5-6, pp. 671-674, 2003. 
[12] G. Dal Negro, M. Bonato, and L. Gribaldo, "In vitro bone marrow granulocyte-macrophage progenitor cultures in the assessment of hematotoxic potential of the new drugs," Cell Biology and Toxicology, vol. 17, no. 2, pp. 95-105, 2001.

[13] R. E. Parchment, M. Gordon, C. K. Grieshaber, C. Sessa, D. Volpe, and M. Ghielmini, "Predicting hematological toxicity (myelosuppression) of cytotoxic drug therapy from in vitro tests," Annals of Oncology, vol. 9, no. 4, pp. 357-364, 1998.

[14] D. Parent-Massin, "Relevance of clonogenic assays in hematotoxicology," Cell Biology and Toxicology, vol. 17, no. 2, pp. 87-94, 2001.

[15] A. Pessina, B. Albella, M. Bayo et al., "Application of the CFUGM assay to predict acute drug-induced neutropenia: an international blind trial to validate a prediction model for the maximum tolerated dose (MTD) of myelosuppressive xenobiotics," Toxicological Sciences, vol. 75, no. 2, pp. 355-367, 2003.

[16] A. Pessina, B. Albella, J. Bueren et al., "Prevalidation of a model for predicting acute neutropenia by colony forming unit granulocyte/macrophage (CFU-GM) assay," Toxicology in Vitro, vol. 15, no. 6, pp. 729-740, 2001.

[17] Z. Lin and Y. Will, "Evaluation of drugs with specific organ toxicities in organ-specific cell lines," Toxicological Sciences, vol. 126, no. 1, pp. 114-127, 2012.

[18] Y. M. Sue, C. Cheng, Y. Chou, C. Chang, P. Lee, and S. Juan, "Ectopic overexpression of haem oxygenase-1 protects kidneys from carboplatin-mediated apoptosis," British Journal of Pharmacology, vol. 162, no. 8, pp. 1716-1730, 2011.

[19] K. Amin, C. Ip, L. Jimenez, C. Tyson, and H. Behrsing, "In vitro detection of differential and cell-specific hepatobiliary toxicity induced by geldanamycin and 17-allylaminogeldanamycin using dog liver slices," Toxicological Sciences, vol. 87, no. 2, pp. 442-450, 2005.

[20] K. Amin, C. Ip, B. Sato et al., "Characterization of ANITinduced toxicity using precision-cut rat and dog liver slices cultured in a dynamic organ roller system," Toxicologic Pathology, vol. 34, no. 6, pp. 776-784, 2006.

[21] H. P. Behrsing, K. Amin, C. Ip, L. Jimenez, and C. A. Tyson, "In vitro detection of differential and cell-specific hepatobiliary toxicity induced by geldanamycin and 17-allylaminogeldanamycin in rats," Toxicology in Vitro, vol. 19, no. 8, pp. 1079-1088, 2005.

[22] H. P. Behrsing, A. E. M. Vickers, and C. A. Tyson, "Extended rat liver slice survival and stability monitored using clinical biomarkers," Biochemical and Biophysical Research Communications, vol. 312, no. 1, pp. 209-213, 2003.

[23] A. E. M. Vickers and R. L. Fisher, "Precision-cut organ slices to investigate target organ injury," Expert Opinion on Drug Metabolism and Toxicology, vol. 1, no. 4, pp. 687-699, 2005.

[24] A. Pessina, A. Bonomi, L. Cavicchini et al., "Prevalidation of the rat CFU-GM assay for in vitro toxicology applications," ATLA Alternatives to Laboratory Animals, vol. 38, no. 2, pp. 105-117, 2010.

[25] Institute for Laboratory Animal Research, Committee for the Update of the Guide for the Care and Use of Laboratory Animals, and National Research Council, Guide for the Care and Use of Laboratory Animals, The National Academies Press, 1996.

[26] G. J. Stevens and C. A. McQueen, "Species variation in the genotoxicity of batracylin," Toxicology and Applied Pharmacology, vol. 126, no. 1, pp. 39-44, 1994. 

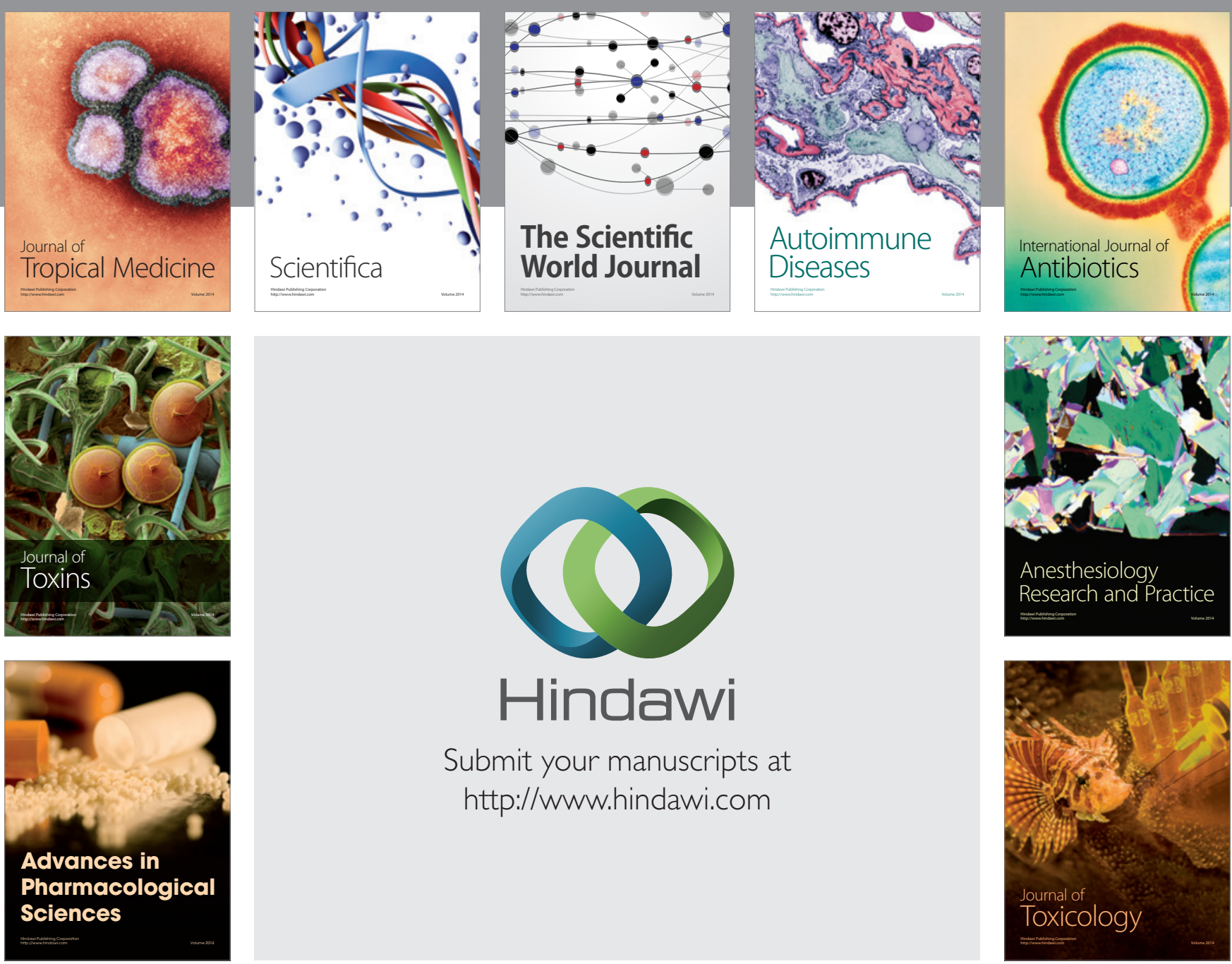

\section{Hindawi}

Submit your manuscripts at

http://www.hindawi.com
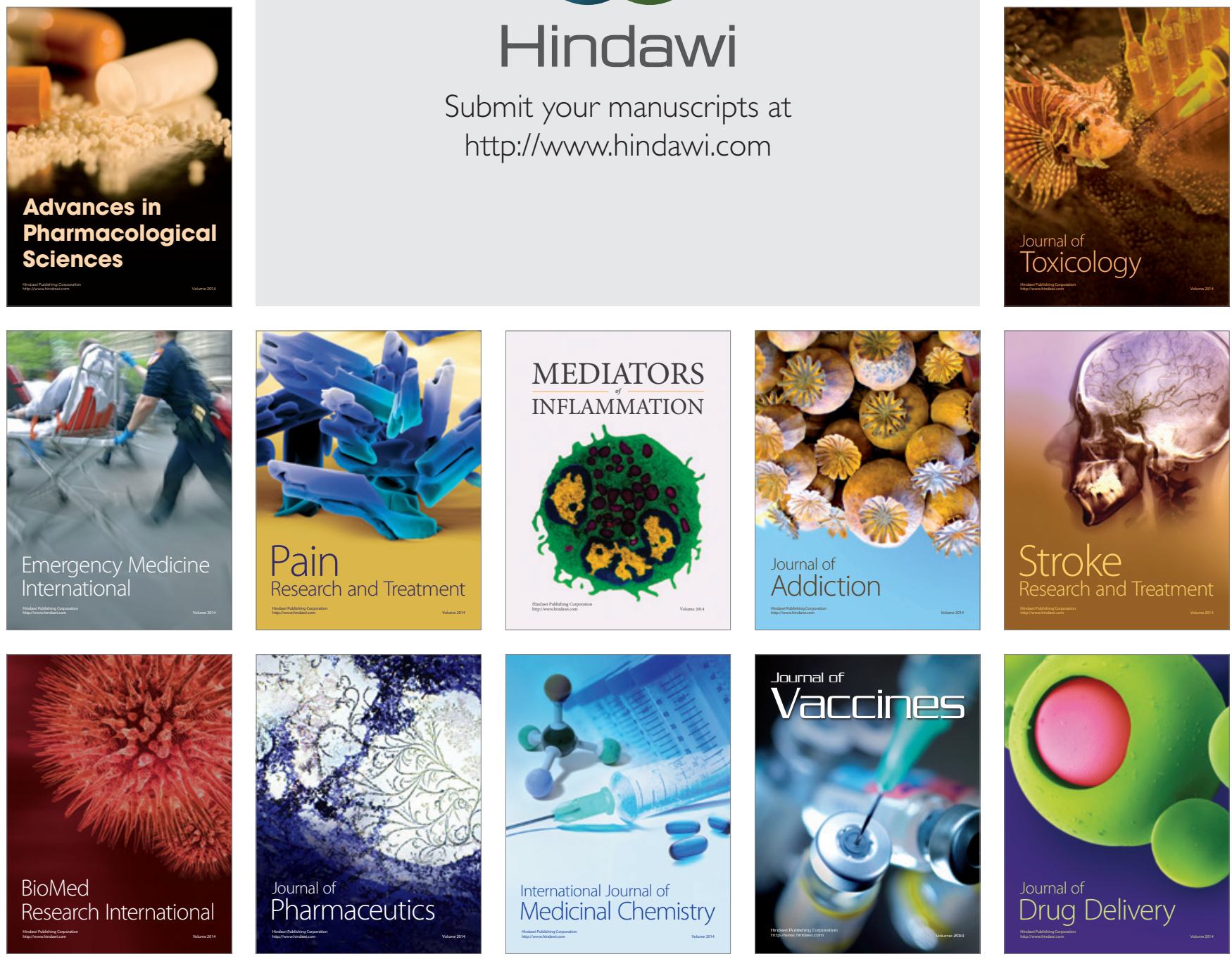